TITLE:

\title{
Propagation of axisymmetric Trivelpiece-Gould mode along vortex columns generated by diocotron instability
}

\author{
AUTHOR(S): \\ Kawai, Y; Kiwamoto, Y; Soga, Y; Aoki, J
}

\section{CITATION:}

Kawai, Y ... [et al]. Propagation of axisymmetric Trivelpiece-Gould mode along vortex columns generated by diocotron instability. PHYSICS OF PLASMAS 2007, 14(10): 102106.

\section{ISSUE DATE:}

2007-10

URL:

http://hdl.handle.net/2433/50223

\section{RIGHT:}

Copyright 2007 American Institute of Physics. This article may be downloaded for personal use only. Any other use requires prior permission of the author and the American Institute of Physics. 


\title{
Propagation of axisymmetric Trivelpiece-Gould mode along vortex columns generated by diocotron instability
}

\author{
Y. Kawai, ${ }^{\text {a) }}$ Y. Kiwamoto, Y. Soga, and J. Aoki \\ Graduate School of Human and Environmental Studies, Kyoto University, \\ Yoshidanihonmatsu-cho-Kyoto-shi, Kyoto-fu 606-8501, Japan
}

(Received 19 June 2007; accepted 12 September 2007; published online 23 October 2007)

\begin{abstract}
A high-frequency (HF) oscillation has been observed to propagate along magnetic field lines with an axisymmetric profile extending over multiple columns of magnetized pure electron plasma which undergo two-dimensional vortex motions. The observed frequency is a few hundred times higher than the rotation frequency of the vortices and varies in time as the density distribution of electrons deforms from an unstable hollow profile into vortex columns and approaches a single-peaked distribution. Experimental examinations reveal that the HF oscillation is excited by the gate pulse applied to prepare the initial profile and that the oscillation does not influence the subsequent dynamics of vortex columns. Further examinations including detailed observations and theoretical analyses indicate that the HF oscillation represents a profile-dependent eigenfunction of the Trivelpiece-Gould mode wave that consists of a dominant axisymmetric component and small fractions of higher azimuthal modes associated with the density distribution separated into columns. (C) 2007 American Institute of Physics. [DOI: 10.1063/1.2793731]
\end{abstract}

\section{INTRODUCTION}

Many aspects of two-dimensional (2D) vortex dynamics have been explored extensively by utilizing properties of the flow composed of guiding centers of strongly magnetized electrons that drift in the direction perpendicular both to the uniform magnetic field $\mathbf{B}=B_{0} \hat{z}$ and to the self-electric field E. ${ }^{1-7}$ The $2 \mathrm{D}$ distribution $n(x, y)$ of electron density is morphologically equivalent to the vorticity of the $2 \mathrm{D}$ flow. ${ }^{7,8}$ Hollow density profiles of electrons are subject to the diocotron instability due to the radial shear of the azimuthal flow. ${ }^{8}$ The dynamics of separated columns of electrons that appear in a nonlinear stage of the instability has been studied to examine fundamental processes of the turbulence in the 2D flow. ${ }^{5-7,9,10}$

The hollow distributions are produced by temporally reducing the barrier height at one end of the square-well potential trap to let electrons near the machine axis escape selectively. The duration of the pulsed operation is to be much shorter than the characteristic time of the vortex dynamics that is evaluated as the $\mathbf{E} \times \mathbf{B}$ drift frequency. For the observation of the time-varying distribution of the electrons, the trapped electrons are fully extracted along the magnetic field line and counted in a 2D array. ${ }^{1-7,9,10}$ This scheme of diagnostics inevitably involves destruction of the electron plasma at each time of the observation and requires repetition of the experimental shots that start from the same initial condition. Because the initial distributions are not free from shot-byshot variations and the subsequent dynamics proceed statistically, there remains uncertainty in understanding the time evolution of the vortex dynamics. In order to compensate this shortcoming, electrostatic signals induced on sectored segments of the conducting wall are recorded as a monitor to compare with the data of the vortex images. ${ }^{2,7,11}$

\footnotetext{
${ }^{a)}$ Electronic mail: kwaiyosuke@h01a0395.mbox.media.kyoto-u.ac.jp
}

The major topics to be reported in this paper have been obtained through extensive examinations of the electrostatic signals over a broad frequency range. The examinations have revealed that high-frequency (HF) oscillations propagate along the magnetic field lines extending radially over vortex columns undergoing stochastic motions. This unexpected observation has stimulated an interest in the origin and nature of the HF oscillations and raised a question about the validity of vortex properties that have been reported without much consideration about potential effects of the HF oscillations.

In this paper, we first present the method of experiments that is closely related to the generation of the HF oscillations, and describe the characteristic features of the oscillations associated with the vortex dynamics. We then address the following two issues related to the oscillations: First, the effect of the oscillations on the vortex dynamics is investigated by comparing the time evolutions of the electron densities in two cases, with and without the oscillations, using a feedback control system. Secondly, the quantitative analysis of the mode identification is carried out in terms of a eigenmode equation including the mode-coupling between the observed asymmetric density distribution and the potential perturbations with various azimuthal modes.

\section{EXPERIMENTAL METHOD}

The dynamics of the pure electron plasma is examined in a Malmberg-trap, as schematically shown in Fig. 1,,11 which consists of a uniform magnetic field $\left(B_{0}=0.048 \mathrm{~T}\right)$ and a square-well potential. The potential distribution is produced by negatively biasing two parts of the electrodes, Nos. 9-11 and $17-19$, at $-90 \mathrm{~V}$ out of 11 ring electrodes axially aligned with a pitch of $24 \mathrm{~mm}$ that form a conducting wall with a radius of $R_{W}=32 \mathrm{~mm}$. Electrons are introduced from preselected cathode elements into the potential well through a temporally decreased potential barrier at the electrode Nos. 


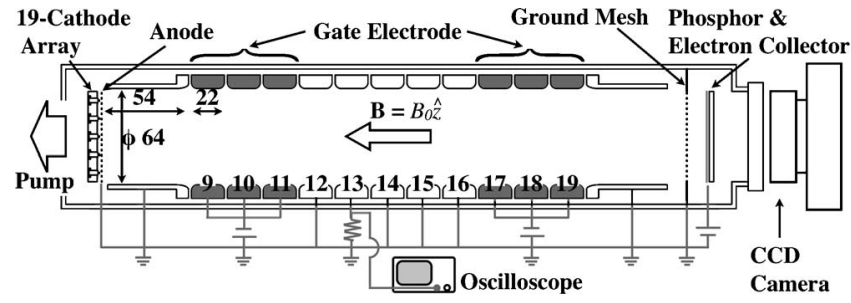

FIG. 1. Schematic view of experimental configuration.

9-11. After about $1 \mathrm{~s}$ of the isolated relaxation of the confined electrons following repetitive operations of an injection-hold cycle, a single-peaked density distribution is established in an axisymmetric form that is axially homogeneous over the trap length of $L \approx 80 \mathrm{~mm}$. Since the plasma potential is the deepest near the axis, electrons in this region are expelled when the potential barrier at the injection side is reduced again in a pulse, and the density profile is shaped hollow.

On the recovery of the bias voltage, the hollow distribution completes the initial configuration of the diocotron instability, and subsequently develops into separated columns in the nonlinear stage of the spontaneous evolution in the trap. ${ }^{6,9,10}$ A set of image data representing a typical evolution of the density distribution is shown in Fig. 2. The distribution at each time step is taken by removing the potential barrier at the electrode Nos. 17-19 and damping the electrons onto the phosphor screen biased at $7.5 \mathrm{kV}$ after the prescribed time of holding. The luminosity distribution on the screen is recorded on the charge-coupled-device $(\mathrm{CCD})$ camera with the dynamic range of 16 bits and $512 \times 512$ pixels providing the spatial resolution of $0.1 \mathrm{~mm} /$ pixel. The surface of the screen is coated by the aluminum film serving as a charge collector to absolutely determine the number of trapped electrons $N_{Q}$ as well as to avoid a charge accumulation on the phosphor surface. A linear relationship between $N_{Q}$ and the integrated luminosity $N_{L}$ of the CCD image has been confirmed, ${ }^{11,12}$ so that the axially integrated $2 \mathrm{D}$ density distribution $n_{l}(x, y)$ is determined absolutely. Based on the axial uniformity of the density distribution, the electron density is evaluated as $n(x, y, z)=n_{l}(x, y) / L$.

An imperturbing monitor is introduced in order to supplement the destructive imaging diagnostics. The dynamics of the electron plasma induce a time-varying chargedensity distribution $\sigma=-\varepsilon_{0} \partial \phi(r, \theta, z) /\left.\partial r\right|_{r=R_{W}}$ on the surface

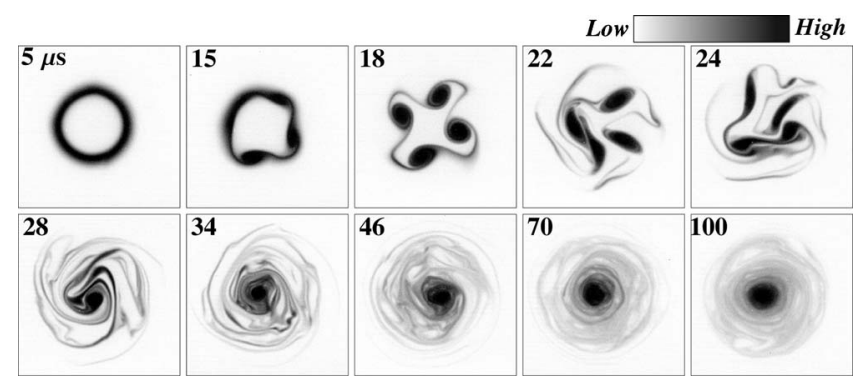

FIG. 2. Images of the time evolution of the density distribution. The darkness is proportional to the density. The time of observation (in microseconds) is indicated at the upper left corner (the density is hollowed at $5 \mu \mathrm{s}$ ).

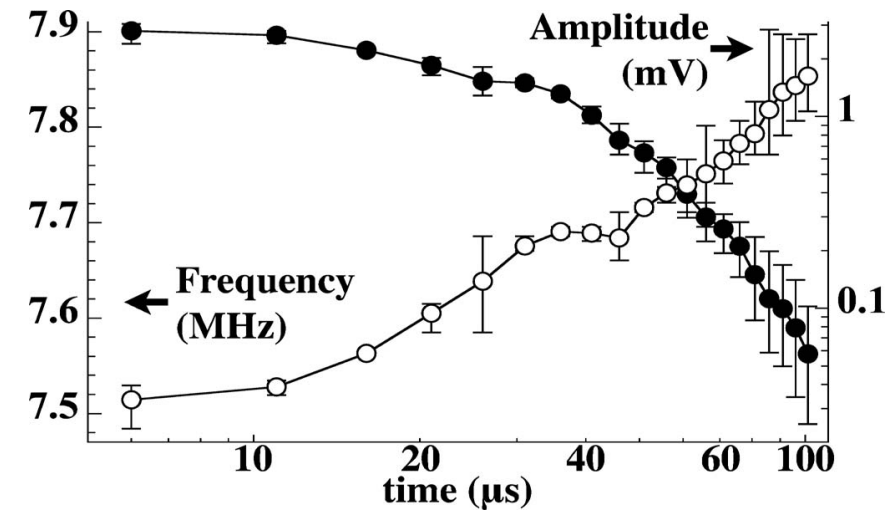

FIG. 3. The time evolution of the amplitude $(\bullet)$ and frequency $(\bigcirc)$ of the HF oscillation observed at the electrode No. 13 in the relaxation process, as shown in Fig. 2.

of the conducting wall in a manner observable without serious disturbances to the plasma. Here, $\phi(r, \theta, z)$ is the selffield potential created by the density distribution $n(r, \theta, z)$, and $\varepsilon_{0}$ is the dielectric constant in vacuum. The spatial distribution of the image charge is detected as currents flowing out of the axially aligned electrode Nos. 12-16, and azimuthal resolution is provided by four $90^{\circ}$ sectors installed on the ring electrode Nos. 12, 14, and 16. The amplitude of a signal $V_{\text {rec }}$ detected on a sector electrode (at the azimuthal position $\theta_{p}$ with the axial length $l=22 \mathrm{~mm}$ and the azimuthal half-width of $\Delta$ ) grounded through a resistance $R(\Omega)$ is related to the amplitude of the time-varying plasma potential $|\phi|$ by the equation ${ }^{11,13}$

$$
V_{\mathrm{rec}}=\varepsilon_{0} R_{W} l R \frac{d}{d t} \int_{\theta_{p}-\Delta}^{\theta_{p}+\Delta} d \theta \frac{\partial \phi\left(R_{W}, \theta, z\right)}{\partial r} .
$$

\section{EXPERIMENTAL RESULTS}

\section{A. Origin and property of the HF oscillation}

The electrostatic diagnostics unexpectedly revealed the appearance of HF oscillations much stronger than lowfrequency signals associated with the vortex dynamics. Experimental examinations focused on the phase difference between the signals detected simultaneously on different electrodes indicate that the HF oscillations are characterized by the azimuthal mode number $m=0$ and the axial wavenumber of $k_{z}=\pi / L$. Figure 3 plots the time evolution of the frequency and amplitude of the HF oscillations observed at the electrode No. 13 in the relaxation process shown in Fig. 2 . The error bars represent the width of shot-by-shot variations. Throughout the whole process, while the wave amplitude undergoes damping, the frequency shows an upward shift. Particularly in the interval from 18 to $34 \mu$ s when the density distribution changes drastically through merger from separated columns down to a single-peaked distribution, the frequency shows a rapid increase and the amplitude decays substantially after this period. It is expected from these observations that the properties of the oscillations are attribut- 


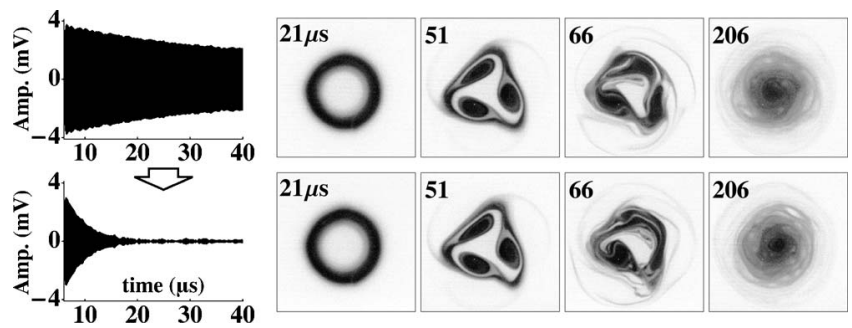

FIG. 4. A comparison between two cases in which the HF oscillations are excited (upper panels) and suppressed (lower panels). The left panels show the detected the HF signals and the right panels display the time evolution of the density distribution.

able to the dispersion relation of body waves propagating in the electron plasma and that the dispersion relies on the timevarying density distribution.

The origin of the HF oscillations is examined by controlling the amplitude and timing of the gating pulse for producing the initial density distribution of a concave profile. Observations indicate that the HF oscillations are excited at the moment when the temporally reduced potential barrier returns to the normal bias of the trap. It is also observed that the HF oscillations are excited even when the decrement of the potential barrier $\Delta V_{\text {plug }}$ is so small that a density profile remains convex. The HF amplitude increases with increasing $\Delta V_{\text {plug. }}$. From these observations, the origin of the HF oscillations is confirmed to be an axial displacement of the plasma column caused by the fast rise of the potential barrier. This consideration indicates that the generation of HF oscillations is not a phenomenon specific to this experimental configuration, but should have been excited in previous vortex experiments. ${ }^{1,2,5-7}$ Actually, Driscoll noticed the generation of high-frequency plasma modes with $k_{z} \neq 0$ and $m=0$ in the hollowing process and examined its contribution to the development of an unstable diocotron mode with $k_{z}=0$ and $m=1$. $^{14}$ Though there is no further description about the identification of the modes, the influence onto the $m=1$ diocotron dynamics was denied on the basis of resistive control of the HF amplitude.

Hereafter, the discussion will focus on (1) the effects of the HF oscillations on the 2D vortex dynamics with timevarying mode number $m$ and (2) the mode identification of the oscillations.

\section{B. Effect of the HF oscillations on vortex dynamics}

The comparison is made between two cases of vortex dynamics, the HF oscillations are excited or suppressed. The suppression of the oscillations is carried out by inserting a negative-feedback circuit between two electrodes at Nos. 13 and 15, by which the HF signal detected at No. 13 is fed to No. 15 after varying its amplitude and phase. Figure 4 shows the detected HF signals and associated density distributions in the two cases. While the HF oscillations are completely suppressed at $20 \mu \mathrm{s}$ in the lower panels, there appear no differences exceeding shot-by-shot variations in the density distribution representing the vortex dynamics.

Quantitative comparisons between the two cases are made in Fig. 5. Here, the vortex structures are characterized

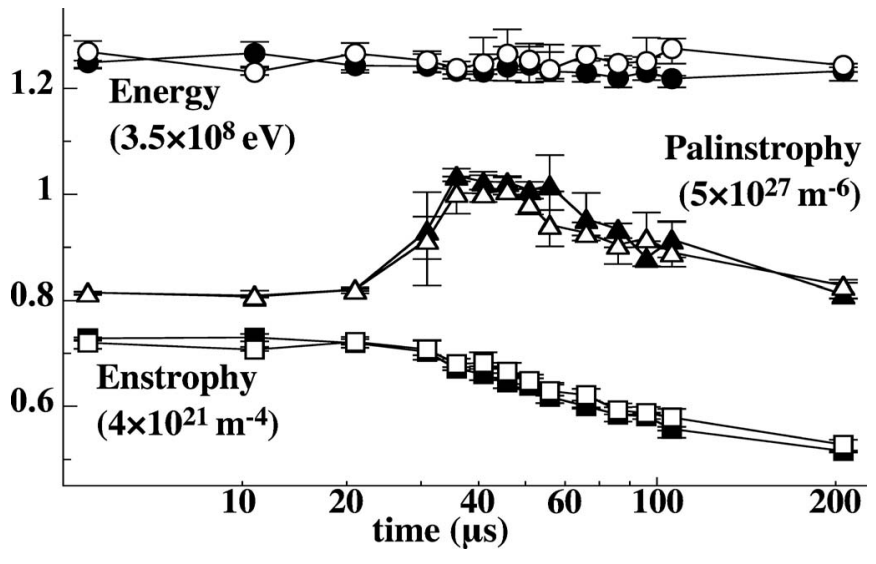

FIG. 5. The time evolution of the energy (circles), enstrophy (squares), and palinstrophy (triangles) with (open symbols) and without the HF oscillations (closed symbols).

in terms of three integrals calculated from the observed density distribution $n(x, y)$, the system energy $E=\frac{1}{2} \int L d^{2} \mathbf{r} n(-e \phi)$, the enstrophy $Z_{2}=\frac{1}{2} \int d^{2} \mathbf{r} n^{2}$, and the palinstrophy $P=\frac{1}{2} \int d^{2} \mathbf{r}|\nabla n|^{2}$. The enstrophy and palinstrophy are a measure of the peakiness and fineness in the spatial structures of the density $n(x, y)$, respectively. ${ }^{10,15,16}$ Figure 5 indicates that each integral is close to its counterpart within the extent of shot-by-shot stochasticity, regardless of the presence of HF oscillations. These analyses support the validity of the present understanding of vortex dynamics and turbulence based on the experimental observations with pure electron plasmas. $1,2,5-7,9,10,14$

\section{Mode identification of HF oscillations}

As stated in Sec. III A, the observed oscillations are axisymmetric $(m=0)$ and have the axial wave-number of $k_{z}$ $\approx \pi / L$ in a frequency range of $\omega_{\mathbf{E} \times \mathbf{B}} \ll \omega_{\mathrm{HF}}<\omega_{p} \ll \omega_{c}$, where $\omega_{\mathbf{E} \times \mathbf{B}}$ and $\omega_{\mathrm{HF}}$ are the characteristic frequency of the vortex motions and HF oscillations, and $\omega_{p}$ and $\omega_{c}$ are the electron plasma and cyclotron frequency, respectively. These properties suggest that the $\mathrm{HF}$ oscillations belong to Trivelpiece-Gould (TG) modes. ${ }^{17}$ However, the identification as an axisymmetric TG mode propagating in irregularly aligned electron columns is not readily acceptable because previous analyses have been concentrated to the case of propagation in a continuously connected density distribution $^{14}$ and in most cases with axisymmetric profile. $^{13,17,18}$

Experimental evidence supporting this identification is that the HF oscillations remain observed in the same frequency range even when the decrement of the potential barrier $\Delta V_{\text {plug }}$ is decreased to such a low level that electrons are not drained to trigger the diocotron instability. The open circles in Fig. 6 represent the frequency of the HF oscillations observed when $\Delta V_{\text {plug }}=60 \mathrm{~V}$. Because the initial profile of the density distribution remains convex, the diocotron instability does not set in, nor do vortex columns appear. The open squares stand for a solution of the eigenmode equation 


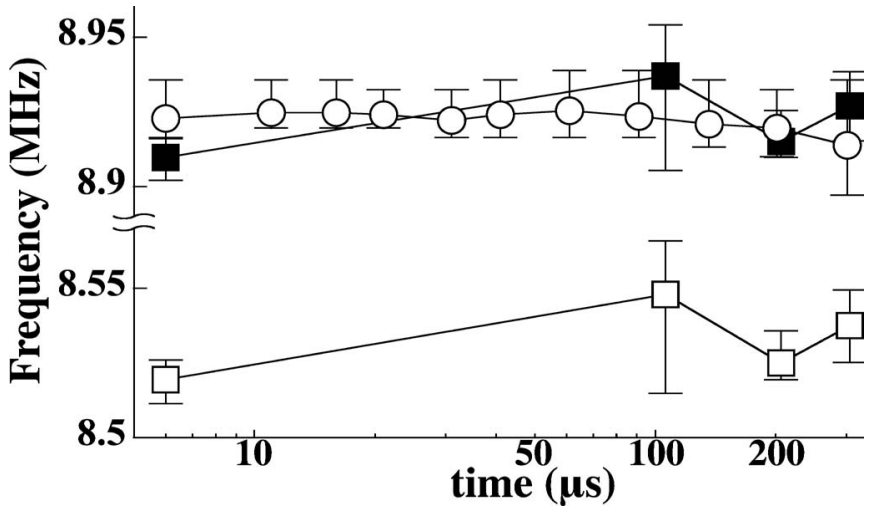

FIG. 6. The time evolution of the frequency measured in the unhollowed densities $(\mathrm{O})$, eigenfrequency $\omega / 2 \pi$ calculated from Eq. (2) for $T_{e}=0 \mathrm{eV}$ $(\square)$, and $\omega_{T} / 2 \pi$ for $T_{e}=0.3 \mathrm{eV}(\square)$

for a potential perturbation $\varphi_{0}(r)$ that belongs to the axisymmetric TG mode propagating in an axisymmetric density distribution $n_{0}(r)^{13,18}$

$$
\frac{1}{r} \frac{d}{d r}\left(r \frac{d \varphi_{0}(r)}{d r}\right)-k_{z}^{2}\left(1-\frac{1}{\omega^{2}} \frac{e^{2}}{\varepsilon_{0} m_{e}} n_{0}(r)\right) \varphi_{0}(r)=0,
$$

where $-e$ and $m_{e}$ are the electron charge and mass, respectively. The boundary conditions are imposed so that $\varphi_{0}\left(R_{W}\right)=0$ and $d \varphi_{0}(0) / d r=0$. The experimental data are introduced for the density distribution $n_{0}(r)$ and $k_{z}=\pi / L$. The electron temperature $T_{e}$ is set to be zero in Eq. (2). The correction of the finite temperature is given approximately by $\omega_{T}^{2}=\omega^{2}+3 T_{e} k_{z}^{2} / m_{e}$. The closed squares represent the frequency corrected for the finite temperature of $T_{e}=0.3 \mathrm{eV}$, a typical electron temperature in the present experiments. A satisfactory agreement between the observed frequency and the eigenfrequency supports the argument that the observed HF oscillations belong to the TG mode exited by the gating pulse imposed to the potential barrier.

\section{DISCUSSION}

On the basis of the above arguments, we extend the mode analysis to the case of asymmetric density distributions as shown in Fig. 2. After expanding the potential perturbation $\delta \phi(r, \theta, z, t)$ and the zeroth-order density distribution $n(r, \theta)$ in Fourier series as $n(r, \theta)=\Sigma_{m^{\prime}} n_{m^{\prime}}(r) e^{i m^{\prime} \theta}$ and $\delta \phi(r, \theta, z, t)=\Sigma_{m} \varphi_{m}(r) e^{-i\left(\omega t-m \theta-k_{z} z\right)}$, the eigenmode equation with azimuthal mode number $m$ is written as

$$
\begin{aligned}
& \frac{1}{r} \frac{d}{d r}\left(r \frac{d \varphi_{m}}{d r}\right)-\frac{m^{2}}{r^{2}} \varphi_{m}-k_{z}^{2}\left(1-\frac{1}{\omega^{2}} \frac{e^{2}}{\varepsilon_{0} m_{e}} n_{0}\right) \varphi_{m} \\
& \quad=k_{z}^{2} \frac{e^{2}}{\varepsilon_{0} m_{e}} \frac{1}{\omega^{2}} \sum_{m^{\prime} \neq m} n_{m-m^{\prime}} \varphi_{m^{\prime}}
\end{aligned}
$$

in the frequency range relevant to the observation. The terms on the left-hand side describes the potential perturbation of the TG mode propagating in an axisymmetric density distribution $n_{0}(r)$, and the right-hand side represents the mode coupling between asymmetric components of density distributions and potential perturbations. The eigenvalue $\omega$ is determined for $k_{z}=\pi / L$ so that the solutions $\varphi_{m}(r)$ to the simul-

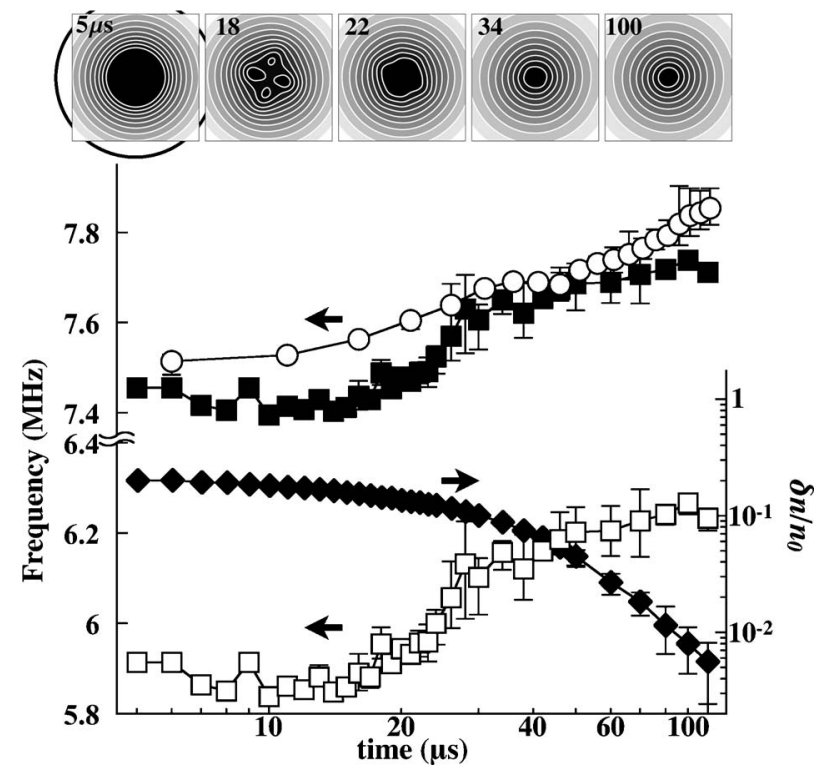

FIG. 7. The time evolutions of $\omega / 2 \pi(\square)$ for $T_{e}=0 \mathrm{eV}$ and $\omega_{T} / 2 \pi$ for $T_{e}$ $=1 \mathrm{eV}(\mathbf{\square})$ obtained from the observed density distributions in Fig. 2, and that of the observed frequency as plotted in Fig. $3(\bigcirc)$ (left vertical scale). The solid diamonds $(\checkmark)$ show the time evolution of the mode amplitude $\delta n / n_{0}$ calculated from Eq. (4) (right vertical scale). Top: The contour plot of the eigenfunctions determined as the solution to Eq. (3) (normalized to unity). The solid arc at the left stands for the wall radius.

taneous equations of Eq. (3) with $m=-m_{\max } \cdots m_{\max }$ satisfy the boundary conditions that $\varphi_{m}\left(R_{W}\right)=0$ for all $m$ and that $\varphi_{m}(0)=0$ for $m \neq 0$ and $d \varphi_{m}(0) / d r=0$ for $m=0$.

The open squares in Fig. 7 stand for the eigenfrequency $\omega / 2 \pi$ calculated from Eq. (3) based on the density distributions observed at each time step as plotted in Fig. 2. Here, the number of the azimuthal modes are taken to be sufficiently large up to 20 . The observed frequency, as already shown in Fig. 3, is plotted again in the open circles for comparison. Though there remains a noticeable frequency gap of about $20 \%$, the general tendency of an upward shift is common to the observed and calculated frequencies.

For quantitative agreement with the experimental results, it is necessary to include the contribution of the finite temperature of $T_{e}=1 \mathrm{eV}$ into the eigenfrequency obtained for $T_{e}=0 \mathrm{eV}$, as shown in the closed squares. Because it is difficult technically to determine the energy spectra ${ }^{19}$ while the density distribution varies in time with substantial stochasticities, the introduction of $T_{e}=1 \mathrm{eV}$ remains as an assumption without directly supporting observational data. However, it is not unreasonable to expect such an amount of temperature in the presence of the turbulent mixing of vortex columns. Actually, in other experiments with the same machine, the electron temperature has been observed to be around $1 \mathrm{eV}$ right after a single-peaked distribution is established after mixing of many electron strings generated through multiple injection from the cathode array.

The eigenfunctions of the TG mode $\delta \phi(r, \theta)$ satisfying Eq. (3) at each time step is plotted at the top of Fig. 7. The absolute values of $\delta \phi$ is determined so that the radial electric fields at the wall $-\partial \delta \phi /\left.\partial r\right|_{r=R_{W}}$ match the experimental data obtained by using Eq. (1) with the measured amplitude of the 
HF oscillation $V_{\text {rec }}{ }^{13,20}$ In Fig. 7, the eigenfunctions are normalized to unity. In association with the clear appearance of four vortex columns in Fig. 2, a quadruple component is reproduced in the theoretically expected frame at $18 \mu \mathrm{s}$. In spite of the appearance of the $m=4$ mode, the spectrum analysis of the mode energy $\propto\left|\varphi_{m}\right|^{2}$ indicates that the fraction of the power of asymmetric modes remains less than $1 \%$ of the total HF power throughout the whole process.

The density perturbation of the TG mode $\delta n(r, \theta)$ is obtained using the relationship with the eigenfunction $\delta \phi(r, \theta)::^{13,20}$

$$
\delta n(r, \theta)=-k_{z}^{2} \frac{e}{m_{e}} \frac{1}{\omega^{2}} n(r, \theta) \delta \phi(r, \theta) .
$$

The time evolution of the amplitude of the TG mode $\delta n / n_{0}$ is shown in Fig. 7 as the solid diamonds. Here, $\delta n$ and $n_{0}$ are the spatially averaged density perturbation and zeroth-order density, respectively. When the mode is excited, the TG mode component occupies about $20 \%$ of the plasma density, and this large amplitude is sustained even when the density distribution is separated into the vortex columns.

\section{CONCLUSION}

From the above analyses, it is concluded that the HF oscillations belong to a body wave that propagates as a TG mode under the dominance of an axisymmetric eigenmode extending over separated electron columns. The propagation of an axisymmetric TG mode astride separated densities has been overlooked in previous studies. Though any contribution of the HF waves to vortex dynamics may be discarded in the context of the observations and interpretations so far re- ported, the observation that the frequency of the HF wave is affected by the distribution of vortex columns may be useful for unperturbing diagnostics.

\section{ACKNOWLEDGMENTS}

This research was supported by the Grant-in-Aid for Scientific Research (B) 17340173 of JSPS and partly by the collaborative program of NIFS.

${ }^{1}$ K. S. Fine, C. F. Driscoll, J. H. Malmberg, and T. B. Mitchell, Phys. Rev. Lett. 67, 588 (1991).

${ }^{2}$ T. B. Mitchell and C. F. Driscoll, Phys. Fluids 8, 1828 (1996).

${ }^{3}$ Y. Kiwamoto, K. Ito, A. Sanpei, and A. Mohri, Phys. Rev. Lett. 85, 3173 (2000).

${ }^{4}$ K. S. Fine, A. S. Cass, W. G. Flynn, and C. F. Driscoll, Phys. Rev. Lett. 75, 3277 (1995)

${ }^{5}$ X.-P. Huang and C. F. Driscoll, Phys. Rev. Lett. 72, 2187 (1994).

${ }^{6}$ A. J. Peurrung and J. Fajans, Phys. Fluids A 5, 493 (1993).

${ }^{7}$ C. F. Driscoll and K. S. Fine, Phys. Fluids B 2, 1359 (1990).

${ }^{8}$ R. H. Levy, Phys. Fluids 8, 1288 (1965).

${ }^{9}$ Y. Kawai, Y. Kiwamoto, K. Ito, A. Sanpei, Y. Soga, J. Aoki, and K. Itoh, J. Phys. Soc. Jpn. 75, 104502 (2006).

${ }^{10}$ Y. Kawai, Y. Kiwamoto, Y. Soga, and J. Aoki, Phys. Rev. E 75, 066404 (2007).

${ }^{11}$ K. Ito, Y. Kiwamoto, and A. Sanpei, Jpn. J. Appl. Phys., Part 1 40, 2558 (2001).

${ }^{12}$ J. Aoki, Y. Kiwamoto, Y. Soga, and A. Sanpei, Jpn. J. Appl. Phys., Part 1 43, 7267 (2004)

${ }^{13}$ Y. Soga, Y. Kiwamoto, and N. Hashizume, Phys. Plasmas 13, 052105 (2006).

${ }^{14}$ C. F. Driscoll, Phys. Rev. Lett. 64, 645 (1990).

${ }^{15}$ G. K. Batchelor, Phys. Fluids 12(Suppl. II), 233 (1969).

${ }^{16}$ U. Frisch, Turbulence (Cambridge University Press, Cambridge, 1995), p. 21.

${ }^{17}$ A. W. Trivelpiece and R. W. Gould, J. Appl. Phys. 30, 1784 (1959).

${ }^{18}$ R. C. Davidson, Physics of Nonneutral Plasmas (Addison-Wesley, Redwood City, CA, 1990), pp. 240-253.

${ }^{19}$ D. L. Eggleston, C. F. Driscoll, B. R. Beck, A. W. Hyatt, and J. H. Malmberg, Phys. Fluids B 4, 3432 (1992).

${ }^{20}$ J. R. Danielson, F. Anderegg, and C. F. Driscoll, Phys. Rev. Lett. 92, 245003 (2004) 